\title{
EL PROGRAMA DE PUTNAM EN LA DISCUSIÓN REALISMO/ANTIRREALISMO. DEL REALISMO INTERNO AL REALISMO PRAGMÁTICO.
}

\section{María Elena Candioti de De Zan*}

Las discusiones acerca del realismo han vuelto a ocupar un lugar central en las reflexiones filosóficas, constituyéndose en uno de los focos en que convergen cuestiones semánticas, ontológicas y epistémicas. Una presentación actual del debate consiste en caracterizar las posiciones realistas/antirrealistas a partir de la relación lenguaje realidad, tomando como eje la "tesis de la independencia" y estableciendo la siguiente oposición:

a) el realismo debe edificarse necesariamente sobre la cosa en sí y sobre la tesis de la "independencia" de lo real. Tal tesis sostiene que hay un conjunto de objetos estructurados por determinadas propiedades (realismo metafísico) ${ }^{1}$,

b) la opción contraria es negar la tesis, pero en este caso debe aceptarse la consecuencia de negar el realismo. Es a partir de allí que se conciben las diversas formas de antirrealismo o irrealismo.

El modo en que se estructura el debate sobre la tesis de la independencia es, sin embargo, discutible, ya que revela la persistencia del dualismo exterior-interior, conduciendo a una serie de polarizaciones que obstaculizan el modo en que ser resuelven las cuestiones acerca de la justificación de la validez de los enunciados. Con esta discusión pretendemos dar lugar a la reflexión sobre el impacto que las posiciones "semanticistas" han tenido en la temática epistemológica. Entendemos que este recorte reductivo, conjugado con la opción de privilegiar el análisis del lenguaje como instancia primaria, es uno de los motivos que ha dado lugar a que la

\footnotetext{
* Profesora de la Fac. de Ciencias de la Educación de la UNER y de la Fac. de Humanidades de la UNL (macandioti@arnet.com.ar).

1 La caracterización del debate realismo/antirrealismo puede verse en Devitt, Michael, Realism and Truth, Blackwell, Oxford UK \&Cambridge USA, 1991 ( $2^{\mathbf{a}}$ ed.).
} 
problemática epistemológica tenga que enfrentarse a caminos muy estrechos, y que la posibilidad de superar el reduccionismo semántico requiere abordar la cuestión de la validez desde una consideración más compleja y dinámica del lenguaje.

Nuestro propósito en este trabajo es analizar los aportes de Putnam a esta discusión, seguirlo en un proceso de desarticulación de supuestos, y explorar hasta dónde puede avanzarse en los caminos por él indicados. Los sucesivos desplazamientos teóricos que el mencionado filósofo ha realizado, pueden interpretarse como el intento de superar las antinomias del realismo, atendiendo especialmente al modo en que éstas se presentan una vez que se ha adoptado el punto de vista lingüístico. El objetivo de su investigación es precisar la posibilidad del lenguaje de "referir" a lo real como aquello que se da de manera independiente, y en caso de admitirlo, si esto se realiza de manera directa o indirecta. En esta formulación se vislumbran ya las paradojas que se suscitan en torno a la idea de independencia y las dificultades para precisar la noción de referencia. En ello queda implicado el modo en que se entienden la verdad y las posibilidades de justificación, por lo cual cada uno de estos corrimientos lo obligan a revisar estas cuestiones.

Intentaremos en primer lugar señalar algunos aspectos definitorios de la posición llamada "realismo interno"; tomaremos para ello como eje la tesis de la "independencia" y el modo en que se articula con la idea de referencia "directa" y de verdad "epistémica".

En segundo lugar, nos detendremos en ciertas ambigüedades relacionadas con aspectos centrales de esta forma de realismo, tratando de mostrar la insuficiencia del recurso a la idea de referencia directa tal como se deriva del análisis de los componentes del significado. La hipótesis que orientará nuestro análisis es que no solamente la idea de referencia directa y los procedimientos señalados en estos textos para su determinación resultan ambiguos, sino que es la perspectiva semanticista que persiste en los trabajos correspondientes a la etapa del "realismo interno" lo que condiciona los términos mismos con que se plantea el problema, obstaculizando la pretendida restitución del realismo.

En tercer término analizaremos las alternativas que se abren en el tránsito que realiza Putnam hacia una nueva forma de realismo. Las ideas de significado y referencia quedan en este caso vinculadas a las de intencionalidad y funciones perceptivas, en un "realismo natural" de fuerte impronta pragmática y 
wittgensteiniana. Finalmente se considerará la potencialidad del programa de Putnam.

\section{El realismo interno}

Las primeras versiones del realismo interno fueron inspiradas por una toma de posición que puede caracterizarse como "antirrealismo global"2. En Razón, Verdad e Historia, se lo presentó como un "verificacionismo moderado" mientras que en Las mil caras del realismo se lo caracterizó por su oposición al realismo metafísico y a los supuestos que lo sostenían. El objetivo era mostrar que tras la disyuntiva que polariza las opciones se encuentran una serie de dicotomías, tales como la de exterior-interior, mentecuerpo, hecho-convención. La estrategia adoptada consistió en poner en evidencia las dificultades de las posiciones externalistas especialmente en lo que concierne a la posibilidad de "representar" una realidad estructurada en $s i^{3}$.

El Realismo (con $\mathrm{R}$ mayúscula), el realismo metafísico, ya sea en sus formas explícitas, o agazapándose tras el realista "científico", tal como ingeniosamente lo presenta Putnam 4 , es lo que le resulta insostenible. Su postulación se apoya en determinados supuestos:

1) Hay una totalidad fija de todos los objetos.

2) Hay una totalidad fija de todas las propiedades.

3) Hay una separación estricta entre las propiedades que "descubrimos" en el mundo y las que "proyectamos" sobre él.

4) Hay una relación fija de correspondencia a partir de la cual puede definirse la verdad.

2 Se sigue en este caso la caracterización realizada por Dumett. Dumett, Michael: Truth and Other Enigms. Londres, Duckworth, 1978.

${ }_{3}^{3}$ Putnam intenta con el realismo interno superar su propio realismo científico y la etapa de "irrealismo" de "Modelos y realidad" en Putnam, H.: Realismo y Razón, Philosophical Papers, vol 3. Cambridge University Press, 1983 y " Realismo y razón”, en Putnam, H: El significado y las ciencias morales, Méjico, UNAM, 1991.

4 Putnam, H.: Las mil caras del realismo, (MCR) Barcelona, Paidós, 1994, p. 42. 
Cada uno de estos supuestos muestra su debilidad en cuanto tomamos conciencia de la intervención del lenguaje. Putnam quiere separarse tanto de esta forma que impone una única descripción de la realidad de manera excluyente, como de un antirrealismo "a lo Rorty", posición que se visualiza como una peligrosa aceptación de la "pérdida del mundo", para poner en su lugar el lenguaje. Opta así por una peculiar forma de realismo que parte de la aceptación de múltiples esquemas lingüísticos conceptuales desde los que conocemos.

En oposición a toda concepción dogmática, el internalismo niega que pueda darse una única manera de decir lo que la realidad es en sí misma, independientemente de cualquier descripción. Lo que queda en primera instancia cuestionado es la "tesis de la independencia". La afirmación de objetos es siempre dependiente o "relativa" al punto de vista. No es posible para el hombre un punto de vista absoluto, algo así como "el punto de vista del Ojo de Dios". Este reconocimiento implica no solamente aceptar la limitación de nuestras perspectivas, sino también que la descripción introduce la mediación de esquemas conceptuales diversos, resultando por lo tanto absurdo bablar de objetos y propiedades que se dan con independencia del lenguaje. No tiene sentido hablar de "cosas en sí" y de signos que las representen a través de una correspondencia intrínseca, independiente de su modo de empleo y de quienes están involucrados en esta descripción; por lo tanto debe hablarse de una dependencia relativa a modelos, teorías, descripciones, etc. ${ }^{5}$

Pese a estas afirmaciones, el realismo interno no se presenta como un inmanentismo o un relativismo. Si bien queda negada la posibilidad de

5 Admitir la intervención humana implica abandonar la pretensión de una única descripción absolutizada. Si queremos encontrar un ejemplo de estas posible múltiples descripciones dentro del ámbito de la ciencia, la interpretación de Copenhagen de la mecánica cuántica resulta ilustrativa. Igual que Kant -sostiene Putnam- Bohr entendió que el mundo en sí estaba más allá de la capacidad cognitiva de la mente para describirlo, pero a diferencia de Kant, advirtió que no hay una única pintura que logre una descripción completa. Más allá de las diferencias de enfoques y de expectativas diversas en la mecánica cuántica en cuanto a superar (o no) esta situación incómoda para quien proviene de la física clásica, lo que aquí se muestra es que el conocimiento implica intervención. 
una única descripción verdadera -tal como lo admitía el realismo metafísicoPutnam está muy lejos de aceptar la idea de que el mundo no es más que una versión lingüística ${ }^{6}$. Por esto, el otro pilar sobre el que se sostiene el realismo interno es el de una de verdad que se distancia tanto de la noción de copia de una realidad externa, como de las concepciones contextualistas. Se elabora así una concepción epistémica de verdad que pretende dar cuenta de los procesos de corrección. En una formulación de clara inspiración kantiana, la verdad queda definida como "bondad última de ajuste". La verdad es una noción normativa, es aceptabilidad racional en condiciones ideales, siendo esta referencia a la "idealidad" de las condiciones lo que permitiría trascender los contextos concretos y evitar el relativismo ${ }^{7}$.

Queda aún por explicar la posibilidad de articulación de estos diferentes marcos en los procesos de corrección y ajuste, de tal modo que no sea simplemente un acuerdo intralingüístico. Para que se den estos procesos hace falta una cierta continuidad y convergencia, lo cual supone la posibilidad de referirse a 10 mismo, aún cuando las formas de representación o descripción sean diversas. El camino que ve Putnam es sostener la posibilidad de que los términos lingǘísticos refieran al objeto de manera directa, más allá de las diversas caracterizaciones. Para ello recurre a una semántica "externalista". Se trata de invertir la tesis fregeana por la cual se consideraba que el sentido (Sinn) es lo que determinaba la referencia (Bedeutung). El razonamiento es el siguiente: si es el sentido lo que determina la referencia, y a la vez resulta que el sentido depende de los diferentes lenguajes en los que un término se encuentra enmarcado, no hay modo de determinar la referencia. Cada lenguaje se cierra en su propio círculo. Si en lugar de ello entendemos que puede identificarse un mismo referente pese a las diferencias en la "intensión" de los términos, los procesos de convergencia, trascendiendo la limitación de los diversos juegos lingüísticos, son admisibles. La noción de significado que perfila Putnam al respecto, incluye diversos componentes (marcadores sintácticos y semánticos,

${ }^{6}$ Razón, Verdad e Historia,( RVH), Madrid, Tecnos, 1988, p. 13.

${ }^{7}$ Con esta concepción epistémica Putnam pretendía salir de las concepciones correspondentistas, pero también de esa forma de empirismo que considera la verdad de un enunciado en función de experiencias sensoriales (verificacionismo). Putnam, H.: Representación y Realidad (RR), Barcelona, Gedisa, 1990, Capítulo 7. 
estereotipo y extensión), los cuales tendrían cierta independencia. Esto daría la posibilidad de que se determinase la referencia sin la intermediación del sentido lingüístico correspondiente a las diversas descripciones. $\mathrm{La}$ insistencia en la idea de referencia directa, apunta a responder a dos cuestiones en que la idea de verdad se ve comprometida: dar cuenta de la posibilidad de comunicación y confrontación, aún considerando distintos lenguajes, y restituir la idea de que el lenguaje originariamente refiere a lo real.

\section{Acerca de las ambigüedades del realismo interno}

No ha sido nuestro propósito exponer de manera pormenorizada el realismo interno, por otra parte ya suficientemente conocido. Hemos recurrido a esta presentación sumaria sólo para realizar algunas consideraciones críticas, que centraremos en tres puntos:

a) la debilidad argumentativa respecto a la tesis de la independencia,

b) los límites de la concepción epistémica de la verdad,

c) las dificultades del internalismo para precisar la noción de "referencia directa"

a) La explícita intención de Putnam de mantener un realismo, se presenta en permanente tensión con la insistencia en los aspectos constructivos del conocimiento. ¿Cómo conjugar la idea de realidad, con la afirmación de que "la mente y el mundo construyen conjuntamente la mente y el mundo"?8.

En las presentaciones del tema se advierte una continua oscilación. Hay expresiones que parecen endurecer la idea de "dependencia": "Los objetos no existen independientemente de los esquemas conceptuales" dice. Pero a la vez, procura separarse de un fenomenismo: "Hay hechos externos; hay una realidad que pretendemos conocer. Lo que no podemos afirmar, porque carecería de sentido, es que los hechos sean independientes

\footnotetext{
${ }^{8}$ RVH, Prefacio. Cfr. p. 212.

${ }^{9}$ RVH., p. 61, p. 63.
} 
de las elecciones conceptuales"10. El acento está puesto aquí en que es la afirmación de la existencia de objetos la que resulta dependiente de los puntos de vista, y no la existencia como tal.

Estas formulaciones encierran una ambigüedad que hace poco consistente la propuesta del realismo interno, o al menos la debilitan ${ }^{11}$. Las dificultades se acrecientan cuando se trata de precisar la noción de objeto. Un punto clave de la propuesta internalista fue renunciar a la exigencia de representar un mundo de objetos que se autoidentifican con independencia de la mente, sin convertir al mundo en una mera proyección subjetivo; pero, las expresiones que Putnam utiliza en Razón, Verdad e Historia, por ejemplo, muestran más el esfuerzo por explicarlo que una teorización acabada. Sostener, por ejemplo, que "el mundo consiste en objetos que se autoidentifican, pero no con absoluta independencia, como lo quiere el externalista"12, no deja de ser desconcertante.

${ }^{10}$ MCR, p. 82.

11 Cabanchik, Samuel, en el artículo titulado "Realismo y relatividad", Revista de Filosofía, $\mathrm{N}^{\circ} 1-2$ (1995) Bs.As., señala "confusiones conceptuales" que dejarían sin sustento al realismo interno. Estas confusiones se derivarían de no distinguir suficientemente las tesis que definen el realismo. Buscando una posible salida a la situación derivada de tales confusiones, Cabanchick sostiene que es posible ver otro sentido de independencia que es compatible con la afirmación de distintos puntos de vista. Se trataría de una independencia relativa; lo "real" podría ser en este caso el "exceso ", lo que queda en los márgenes de cada punto de vista. De este modo, el autor pretende ubicarse en una posición diferente de la de Putnam ( p. 41-42) Al respecto, creemos que Putnam termina postulando esto (una independencia relativa), aunque tal vez no entienda de la misma forma el "exceso". Este señalamiento indica que la idea de "exceso", más que una posible solución al problema del realismo, significa la apertura de un nuevo campo de interrogantes. La evolución del pensamiento de Putnam, (posterior al mencionado artículo publicado por Cabanchick en 1995) muestra interesentes aportes en orden a superar las dificultades del realismo interno. Esto es lo que nos proponemos explicitar en la última parte de este trabajo.

${ }^{12} \mathrm{El}$ texto que transcribimos muestra estas dificultades: 'Yo diría- sostiene- que el mundo si consiste en Objetos que se Autoidentifican en un sentido, pero en un sentido no asequible al externalista. $\mathrm{Si}$, como mantengo, los propios objetos son tanto construidos como descubiertos, son tanto producto de nuestra invención como del factor objetivo de la experiencia, el factor independiente de nuestra 
La perspectiva internalista se hace difícil de sostener. Los supuestos dicotómicos, pese a la intención de Putnam de desembarazarse de ellos, siguen operando y este condicionamiento es lo que le impide superar lo que él mismo ha calificado como el problema de Berkeley.

b) Esta dificultad se traslada de manera inmediata a la noción de verdad. La idea de convergencia asociada a un internalismo con las características señaladas en el punto anterior, parece reforzar un "coherentismo", de tal modo que no es posible distinguir con claridad verdad y justificación. Putnam insiste, sin embargo, en que no deben confundirse.

Al definir la verdad como "aceptabilidad ideal en condiciones ideales", se pretende trascender las condiciones fácticas de aceptación, pero tal pretensión se ve obstaculizada por lo problemático que resulta precisar qué se entiende por condiciones "ideales". Putnam no está dispuesto a aceptar que se comprendan en conexión con un proceso convergente hacia un estado epistémico en el que una comunidad ideal pudiese determinar las condiciones de verdad. Pero al reformularlo como "condiciones óptimas" no tiene ya modo de marcar las diferencias con aquellas posiciones contextualistas de las cuales querían distanciarse ${ }^{13}$.

Por otra parte, la intención de salir del verificacionismo, aduciendo que la noción de "condiciones epistémicas suficientemente buenas" incorpora de algún modo el mundo ${ }^{14}$, se ve entorpecida por la complejidad con que se presenta el tema de la referencia. Con esto nos introducimos en la siguiente cuestión.

voluntad, entonces los objetos pertenencen intrínsecamente a ciertas etiquetas; porque esas etiquetas son las herramientas que usamos para construir una versión del mundo en la que tales objetos ocupan un lugar preminente. Pero este tipo de Objetos que se Autoidentifican no es independiente de la mente; y lo que el externalista quiere es concebir el mundo como si consistiese de objetos que son independientes de la mente, y que al mismo tiempo se autoidentifican. Y esto es lo que no se puede hacer." RVH, p. 63.

${ }^{13}$ Nos hemos referida a esta cuestión en "Los límites de una concepción puramente epistémica de la verdad”. Congreso Nacional de Filosofía, Salta, 2001.

${ }^{14}$ RR, Cap. 7. 
c) Como hemos visto, el proyecto de un realismo interno estaba directamente vinculado a la posibilidad de dar cuenta de cómo el lenguaje refiere a lo real, a la vez que se explicaba cómo pueden darse procesos convergentes a pesar de la diversidad de descripciones. Como vimos, las expectativas de Putnam estaban puestas en la idea de referencia directa.

$\mathrm{El}$ análisis de la concepción del significado que pertenece a la etapa del realismo interno nos enfrenta, sin embargo, a nuevas dificultades. Recordemos que uno de los objetivos centrales de este período es salvar la inconmensurabilidad y los consiguientes relativismos que se derivan de una concepción semántica, en la cual se hace depender la determinación de la referencia de la intensión de los términos. En contraposición Putnam sostiene que no son ni la intensión de los términos ni las representaciones de los hablantes los que fijan la extensión, sino que hay que atender a la distribución del trabajo lingüístico y al "entorno". En la composición del significado habría entonces un elemento indexical que permitiría decir que no es la intensión lo que determina la referencia, sino que estos componentes mantienen cierta independencia. La determinación de la referencia no se ve así transformada por nuestras creencias. "La extensión de 'oro' no ha cambiado en 2000 años, afirma, entendiendo que de este modo evita la inconmensurabilidad.

Este componente indexical tiene que sustentarse en ciertos presupuestos empíricos. Ahora bien: ¿cuáles son las condiciones de la experiencia que nos permiten decir que se trata de lo mismo? ¿Qué presupuestos empíricos son los que dan tal sustento para que la relación de mismidad se acepte? Más aún ¿qué puede admitirse como condición necesaria y suficiente pera precisar la extensión? Para hablar de la misma "extensión" tendríamos que apoyarnos en ciertas relaciones de similitud que nos autoricen a decir, por ejemplo, que dos muestras de metal, pertenecen al "mismo" metal ${ }^{15}$. Aquí es donde pareciera presentarse la disyuntiva: o hay un dominio de objetos, fijo e independiente de las posibles versiones del mundo, que permite fijar la extensión de los términos; o renunciamos a admitir tal totalidad y la existencia de propiedades en sí de los objetos que

15 Putnam, H. "El significado del significado", en Valdez Villanueva, L. (Ed.), La buisqueda del significado. Lecturas de Filosofia del lenguaje, Tecnos, Univ. De Murcia, 1991, p. 142. 
determinen la extensión, y reconocemos las mediaciones lingüísticas. En este caso, si es el lenguaje el determinante, no tenemos más instancias que las convencionales. Y si lo que permite que algo se identifique como "lo mismo" no tiene otro apoyo que el hecho de compartir ciertas "practicas lingüísticas", resulta muy difícil poder explicar cómo evitar el paso por la "intensión". La determinación de la referencia parece no poder liberarse de la dependencia de los marcos conceptuales y lingüísticos, envolviendo todo el planteo en una cierta circularidad ${ }^{16}$.

Al menos en estos textos, Putnam no logra mostrar de manera convincente la posibilidad de una referencia directa y esto es un indicio más de la debilidad del realismo interno. Los caminos laberínticos en que se introduce el análisis nos hacen sospechar que no es la noción de significado de por sí lo que resulta problemático, sino básicamente el modo en que se articulan lenguaje y realidad en un planteo que, aunque advierte las limitaciones derivadas de las posiciones dualistas, no puede desentrañar totalmente aquellos "nichos" en los cuales persisten.

La determinación de la referencia no puede estar sujeta totalmente a hábitos lingüísticos y a un "atrincheramiento" de creencias (en el sentido de Goodman). El hábito lingüístico no puede ser la definición final ${ }^{17}$. Ni la determinación de la referencia ni la inserción en determinado esquema conceptual, es un asunto de "decisión". Si bien podemos admitir que esta determinación está sujeta a intereses y necesidades, no es la simple convencionalidad. Es bastante más de fondo y tiene que ver con la relación del hombre con el mundo.

${ }^{16}$ En un artículo denominado "El nuevo enigma de la inducción y los términos de clase natural", Crítica. Revista bispanoamericana de Filosofia, Vol 34, N 100, abril 2002, Ignacio Avila Cañamares considera que esta dependencia de las creencias para la determinación de la referencia, y la imposibilidad de dar cuenta de la referencia directa, muestra que en este caso prima un trasfondo fregeano (p. 73). La crítica identifica "intensión" con las creencias de los hablantes y compromisos teóricos ( $p$. 79). Disentimos respecto a esta identificación que pondría a Frege en una posición psicologista, o más aún sociologista. Sin embargo, coincidimos en la persistencia de un trasfondo fregeano, dado por el lugar concedido a los análisis semánticos.

17 Faria, Paulo "Deconstrucción de la irrealidad", Dianonia, Vol XLVII, n ${ }^{\circ}$, noviembre 2002, p. 152. 
La cuestión -insistimos- es si el punto de partida adoptado, permite dar cuenta de la relación lenguaje-realidad. Si es posible partir de un análisis del significado como el expuesto, para realizar luego el "tránsito" hacia el mundo. La dificultad no se subsana solamente recurriendo a una noción nueva de significado, tal como aparece en los ya clásicos textos de The Meaning of Meaning. Este es el momento en que se puede comenzar a sospechar de un cierto lastre fregeano. La opción por el análisis de los enunciados lingüísticos como ámbito prioritario, comienza a mostrar sus deficiencias. Si a esto se le agrega una concepción de la relación lenguajerealidad que no logra apartarse del todo de los esquemas dualistas, el círculo se cierra sobre sí mismo.

Putnam reconoce actualmente haberse extraviado en este empeño, y que era insuficiente la idea de "referencia directa", ya que la propuesta no era más que una reforma superficial, una cobertura lingüística que exigía conectar luego los enunciados con determinados datos por medio de restricciones operacionales. Toma conciencia también de que en ella se reiteraban los viejos problemas suscitados por la necesidad de establecer los nexos entre instancias separadas. Pero no pensemos que este reconocimiento le hace abandonar el núcleo de sus intuiciones fundamentales; más bien lo que intenta es salvar las limitaciones descubiertas, planteando de manera diferente la relación conocimiento-lenguaje-realidad. Intentaremos evaluar los avances realizados en esta dirección.

\section{E1 tránsito al realismo pragmático}

En trabajos recientes, tales como los contenidos en La trenza de tres cabos o en Words and Life, ha admitido también que la expresión "realismo interno" es en realidad una etiqueta que confunde ${ }^{18}$, y prefiere hablar de un realismo pragmático o "directo". El paso a esta forma de realismo, que también llama "natural" está dado por la recuperación del "sentido común", en una especie de "segunda ingenuidad"19. Podemos entender esto como la

-18 Putnam, H.: La trenza de tres cabos (TTC), Madrid, Siglo XXI, 2001, p. 215.

${ }^{19}$ Este realismo está inspirado en ese caso en Witttgenstein, apartándose de aquellas interpretaciones de este filósofo que lo ven como inspirador de una nueva forma de 
intención reflexiva -no desprevenida- de atender a las intuiciones básicas acerca del mundo, concebido como un mundo compartido en el que ejercitamos nuestras prácticas e interacciones. Implica, además, el reconocimiento de un nivel pre-epistémico en el que se generan sentidos diversos.

El realismo no es en este caso una concepción filosófica que demuestre la realidad del mundo o pretenda derivarla del análisis del lenguaje, sino un posicionamiento de base. Se trata más bien de una actitud realista, o de una hipótesis presupuesta; de una especie de "anticipación" del horizonte en el que se desarrolla nuestro conocimiento. En efecto, ¿habría conocimiento y lenguaje sin la "anticipación" de un mundo compartido?

En "The question of realism", Putnam insiste en que la realidad del mundo no puede probarse; es absurdo pensar que podamos tener por un lado el lenguaje y por otro lado el mundo, mirando desde un punto de vista exterior y compararlos. Sin embargo, de ello no se sigue -aclara- que el lenguaje y el pensamiento no refieran a algo que está más allá de ellos mismos, aunque sólo podamos hablar de ese algo describiéndolo. Sostener un realismo no quiere decir entonces acceder al mundo tal como es en sí mismo despojado de toda determinación de pensamiento y lenguaje ${ }^{20}$. La "tesis de la independencia" de un mundo en sí no tiene sentido, como tampoco tiene sentido su negación.

La renuncia de Putnam respecto a la metafísica es explícita. Más aún, a partir de allí argumenta contra posiciones como la de Rorty, denunciando su incoherencia, y mostrando que este último al negar la posibilidad de representación y descalificar la posibilidad de un realismo, toma paradójicamente una posición metafísica. En su radicalidad, Rorty ha caído en una trampa, ya que es tan ininteligible hablar de un mundo en sí, como su negación ${ }^{21}$.

antirrealismo (a esta orientación han contribuido Kripke, Rorty y Dummett). Sobre realismo natural, directo: TTC, p. 12 a 15.

20 Putnam, H. : "The Question of Realism", en Words and Life, (WL) Ed. Conant, Harvard University Press, 1995, p. 297.

${ }^{21}$ Ibid. p. 299. 
A pesar de estas declaraciones, Putnam no se desprende de ciertas formulaciones que involucran la idea de independencia. En "algún sentido de independencia"-dice-el mundo es independiente del modo en que hablamos, aunque las descripciones que sobre él se hagan sean múltiples y refieran de distinta forma a lo que se presenta de tal o cual manera. Con esto reitera las tesis básicas del realismo de la etapa anterior referidas a la multiplicidad de esquemas conceptuales y marcos de experiencia, aunque lo que aquí cobra fuerza es la idea de que son diversas descripciones de lo real. En esto es necesario evitar confusiones, sostiene enfatizando determinados aspectos de las viejas fórmulas. Son las aseveraciones (no las proposiciones) lo que hacen verdadero o falso que "el cielo sea azul", por ejemplo. Componemos muchas palabras y usos de las mismas, para describir la realidad; y hasta los sentidos en que las diversas sentencias "acuerdan" con la realidad pueden ser varios. Pero no bay verdad si no bay aseveración, sino hay reconocimiento de que el enunciado expresa alguna forma de “acuerdo". Las descripciones pueden ser más o menos exactas, pueden ser formalizadas, simbólicos, selectivas, etc., pero la verdad depende de lo que se da en la realidad.. La sentencia: "Yo tomé café esta mañana", depende de lo que sucedió esta mañana ${ }^{22}$.

Vemos así que se admite la existencia del mundo, aunque no resulta sencillo precisar de qué consta: ¿de cosas, de objetos, de “estados de cosas"? Hay una posible interpretación que Putnam se ocupa de descartar: la duplicación de las estructuras lingüísticas en la realidad. Se opone así a toda forma de "ontologización" del orden gramatical. La idea de que haya algo así como "estados de cosas" que hacen verdaderas a nuestras sentencias, le resulta inaceptable. Esto no implica negar que los objetos a veces hacen verdaderas a nuestras sentencias; lo que se niega es que las sentencias verdaderas correspondan a los llamados "estados de cuestión", dándoles estatuto de objeto. Esto sería inflar nuestra ontología con una especie de doble espectral de las sentencias del gramático. La sentencia "no hay un elefante en esta habitación" es verdadera, -aclara- pero no necesito inventar

22 Ibid., p. 302. En TTC, sostiene: "Coincido con el crítico (realista) en que el mundo es como es con independencia de los intereses de quien describa" ( p. 7). Sin embargo excluye "fantasías metafísicas." No hay una totalidad de Formas, Universales o "propiedades". También se arrepiente de haber hablado (como lo hizo en RVH) de "dependencia" de la mente, en conexión con estos temas TTC, p. 210. 
un estado de cuestión tal como "la ausencia del elefante en esta habitación." No puede pensarse una especie de correspondencia entre las sentencias y una estructura fija del mundo formada según estas sentencias.

Sin embargo, no duda en sostener que una afirmación de conocimiento es una respuesta a la realidad, y en la mayoría de los casos, esto significa una realidad independiente del hablante; aunque la reflexión sobre la experiencia humana sugiere que ni la forma de toda afirmación de conocimiento, ni las diversas maneras en que ellas son respuestas a la realidad, estén fijadas de antemano. Una sentencia es verdadera si ciertos casos o eventos satisfacen las condiciones descritas por las sentencias, condiciones que dependen sobre todo de la progresiva actividad de usar y reformar el lenguaje $^{23}$. Lo que importa es la responsabilidad cognitiva para hacer justicia a lo que describamos. La realidad no dicta la totalidad de las descripciones de una vez y para siempre, sino que constantemente añadimos y modificamos las maneras en las que el lenguaje se hace cargo de la realidad, continuamente renegociamos nuestra idea de la misma. Este "hacerse cargo" de la realidad por parte del lenguaje, despliega un nuevo campo para la filosofía. Desde esta óptica, lo que verdaderamente interesa es la forma en que puede ampliarse la capacidad de nuestros conceptos y la flexibilidad para aceptar nuevos usos del lenguaje.

¿Significa esto el abandono del realismo "interno"? Muchas de sus ideas centrales acerca del realismo se mantienen aunque explicadas ahora de otra forma. Sin embargo, no parece adecuado ya hablar de una perspectiva "internalista", especialmente en cuanto a las connotaciones que esto tiene respecto al modo de entender el conocimiento ${ }^{24}$.

Es fácil advertir que en este corrimiento y ajuste de posiciones también quedan directamente involucradas las ideas de verdad y de referencia a lo real. La suerte del realismo está ligada a ellas, y Putnam se ha ocupado de revisarlas y reajustarlas en cada uno de sus desplazamientos. Así como se esforzó por marcar sus diferencias con el externalismo, lo que ahora quiere dejar en claro es su distanciamiento de aquellas posiciones que centralizan el problema en las condiciones de aserción de las oraciones, como se ve en el

\footnotetext{
23 WL, p. 301.

24 TTC, p. 69.
} 
caso de Dummet. Las derivaciones de una posición como la de Dummet, conducen a situaciones inconcebibles para el sentido común, como la de plantearse como podemos verificar abora, cómo fue el pasado, y hablar en consecuencia de la irrealidad del mismo.

Una cosa es la discusión de la idea platonizante de que el pensar puede ser una actividad autónoma respecto al lenguaje; pero el otro extremo es pensar en el lenguaje como algo que luego tenemos que conectar con lo real. Putnam trata de evitar lo que ve como un sesgo idealista, y su atención recae nuevamente en las condiciones de aceptabilidad. El recurso a "condiciones epistémicas óptimas" (tal como se las concibió en el realismo interno) fue ya un intento de sustraer la cuestión del ámbito exclusivo del análisis del lenguaje y de las condiciones de verificación que el hablante puede efectivamente establecer, entendiendo que de esta forma se involucraba el mundo. Reconociendo que los procesos de verificación suponen un progresivo ajuste, se admitía también que siempre hay un "excedente" respecto a las justificaciones de hecho. Pero, como hemos visto, esto no resultó suficiente ya que quedaba sin responder de manera satisfactoria cómo podemos tener "acceso referencial" al mundo y cómo puede determinarse cuándo las condiciones de aceptación son suficientemente buenas.

La intención de dar lugar a una de las intuiciones básicas del realismo, concediendo al mundo un rol determinante, se vio frustrada en la medida en que la situación epistémica se concebía según las categorías tradicionales. El problema a resolver seguía siendo cómo conectar el sujeto epistémico con la realidad alli "afuera" a través de mediaciones. Putnam reflexiona sobre su propia posición, tratando de desatar los "nudos" que le impedían avanzar en su desarrollo, y descubre que uno de estos nudos fue una concepción de conocimiento que se mantenía aún pendiente de la idea de "intermediarios" 25 .

25 "En mi imagen alternativa (en tanto opuesta a la de Dumett) al mundo se le permitía determinar si yo estaba realmente en una situación epistémica suficientemente buena, o si solamente me parecía estarlo -reteniendo de este modo una importante idea del realismo del sentido común- pero la concepción de una situación epistémica seguía siendo, en el fondo, exactamente la concepción epistemológica tradicional. Mi imagen aún retenía la premisa básica de una interfaz entre el sujeto epistémico y todo "lo de afuera". Pero mientras que la necesidad de 
Si ese es el escollo de base, la solución no puede estar en la articulación de elementos del realismo con el idealismo, sino en un desplazamiento que evite la trampa de las aninomias modificando el modo de entender la experiencia, de tal forma que pueda darse cuenta del "acceso referencial" al mundo. Putnam ve como única salida superar la concepción del conocimiento sensorial de la modernidad. Desde el siglo XVII la filosofía ha oscilado entre posiciones impracticables, trabadas por el empeño de explicar el conocimiento en términos causales reduciendo la experiencia a recepción de datos. Una vez que se acepta que las "impresiones" sensoriales son efectos, afecciones de un sujeto, y que estas impresiones se interponen en nuestra relación con el mundo, el camino hacia el idealismo parece ya trazado. A esto hay que agregar la dificultad para explicar el conocimiento en términos puramente causales.

¿Cómo sostener la exigencia de referencia directa y a la vez arrastrar tal concepción del conocimiento? Recordemos que este es el punto clave: ¿cómo atender en la determinación de la referencia a la contribución del "entorno", si no es sobre la base de lo que la experiencia capta de la realidad? La posibilidad de dar cuenta de este "acceso referencial" al mundo le obliga a precisar cómo se entiende en este caso la verdad en su conexión con los procesos de corrección, sin que en ello intervenga la noción de representación de lo real, entendida como "copia". La tarea que aparece entonces como prioritaria es explicitar cómo se articulan la experiencia perceptiva con las prácticas lingüisticas y qué quiere decir, en definitiva, el "hacerse cargo" de la realidad.

La recuperación de la idea de percepción no se circunscribe a poner en el eje de discusión una cuestión que en la filosofía analítica había permanecido relegada en las últimas décadas, sino que requiere también

una "tercera vía"- más allá del realismo moderno temprano y del idealismo de Dumett- es algo que siento con la misma intensidad de siempre, dicho tercer camino, como Mc Dowell ha insistido reiteradamente, corta de raíz la idea de que hay una "antinomia" en vez de simplemente pegar elementos del realismo moderno temprano y elementos de la imagen idealista. Ninguna concepción que retenga algo como la noción tradicional del dato sensorial, puede proporcionarnos una salida: una concepción tal necesariamente debe dejarnos, al final, frente a lo que parece un problema insoluble.’TTC, p. 69. 
lograr una nueva comprensión. Esta renovación implica abandonar la concepción causalista y la correspondiente idea de que los datos sensoriales son afecciones de nuestra subjetividad, dadas en la interioridad de la mente, para afirmar que las cosas se pueden experimentar. En este caso, experimentar las cosas no quiere decir recibir ciertos "qualia" a partir de los cuales se componen objetos. Al respecto advierte Putnam: "Con frecuencia se desatiende, si no es que se niega, que parte del contenido de nuestras experiencias visuales conceptualizadas consiste en que uno ve un aspecto particular o una perspectiva de las cosas en cuestión. Contrariamente a una tradición que va desde Hume hasta la Aufbau de Carnap, cuando cierro mis ojos no tengo que inferir que la puerta amarilla ha desaparecido de mi existencia. Experimento mi ver la puerta, justamente como eso, mi ver la puerta, no como la "existencia de una cualidad visual" y experimento el cierre de mis ojos como mi propia acción, una manera personal de evitar ver la puerta, no como algún qualia que dejara de existir"26.

La percepción no es recepción de datos, descubiertos en el sujeto, sino desde el inicio, es un encuentro con un mundo público; y en este encuentro está comprometida la determinada posición de quien conoce y también su acción, de tal modo que el mundo aparece con determinados sentidos. Así entendida, la percepción tiene un carácter relacional. Las experiencias sensoriales no son afecciones pasivas de un objeto llamado "mente", sino en su mayor parte experiencias de aspectos del mundo experimentadas por un ser viviente" ${ }^{27}$. Por eso la percepción no puede entenderse desde el interiorismo de la mente concebida en su inmaterialidad, ni tampoco desde esa nueva forma de reduccionismo dada por la explicación de los procesos cognoscitivos identificando los estados mentales con estados físicos, biológicos, o computacionales. La percepción requiere el ejercicio de capacidades múltiples. Y si bien esto involucta tanto las "transacciones" que establecemos con el entorno como diversas actividades cerebrales, no puede explicarse utilizando el vocabulario causalista propio de las ciencias. Justamente por ello, la reformulación

${ }^{26}$ TTC, p. 184.

${ }^{27} \mathrm{La}$ idea de percepción como ejercicio de capacidades se expone en WL, p. 288Sobre la revisión de la idea de mente ver los trabajos de Words and Life, "How old is the Mind?, "Changing Aristotle's Mind “y "Aristotle after Wittgenstein". 
propuesta exige aceptar una pluralidad de recursos conceptuales y de vocabularios diferentes, no mutuamente reductibles ${ }^{28}$.

La consideración de la percepción como el ejercicio de capacidades múltiples, implica además romper con la idea de una especie de separación de los niveles cognoscitivos que daría lugar a admitir de manera fragmentaria un nivel sensorial, un nivel conceptual y una "posición" de existencia que nos conecta al mundo exterior. En La trenza de tres cabos Putnam trata de mostrar que el camino hacia el realismo pasa por evitar tal fragmentación; no experimentamos el lenguaje como un conjunto de "marcas y ruidos" en cuyo seno tenemos que leer un significado. "Cuando oímos una oración en un lenguaje que comprendemos, no asociamos un sentido con el diseño de determinado signo, percibimos el sentido en el diseño del signo-aclara Putnam-. Las oraciones que pensamos, o incluso las oraciones que oímos o leemos, se refieren simplemente a cualquiera de las cosas sobre las que ellas hablan; no porque las "marcas y ruidos" que veo y oigo ( u "oigo" en mi cabeza, en el caso de mis propios pensamientos) tengan intrínsecamente el significado que tienen, sino porque la oración en su uso no es simplemente un haz de "marcas y ruidos" 29 .

${ }^{28}$ TTC, p. 47. En los últimos años, Putnam ha dedicado sus escritos especialmente a la discusión con el funcionalismo, es decir, al intento de reducir todo el discurso intencional a física más términos computacionales. TTC, p. 43. Este proyecto supone identificar la intencionalidad con nuestros pensamientos, concebidos como una suerte de escritura interior, con su co-variación causal. Lo que critica del funcionalismo es lo que llama tesis de la identidad. "No podemos conseguir que la noción no explicada de 'identidad' de los datos sensoriales con 'estados del cerebro caracterizados funcionalmente', se precise con la ayuda del concepto de reducción de una teoría a otra, si no tenemos idea de la naturaleza de la teoría que suponemos que actía como reductora de la otra (y solamente tenemos una idea vaga y problemática de la teoría que supuestamente tratamos de reducir' (p. 45). La teoría que tratamos de reducir aparece sumamente borrosa (la de los datos sensoriales) y edificada sobre una base dudosa. La cuestión es que estas posiciones acerca de la identidad, se presentan a sí mismas como la forma de terminar con los dualismos. Putnam insiste, sin embargo, en que el abandono de la teoría de la identidad no nos compromete con el dualismo. ${ }_{29}$ TTC, p. 57. Con estas consideraciones Putnam realiza una extensión del "ver como" wittgensteiniano. Siguiendo el famoso ejemplo de Wittgenstein acerca de la captación de un dibujo como pato o como conejo, pone en evidencia no sólo el 
Lo que especialmente interesa destacar en este párrafo, es que las oraciones se refieren a las cosas de las cuales hablan. $\mathrm{Y}$ esto no tiene porqué despertar sospechas de platonismo, ya que no se afirma que tengan intrínsecamente determinados significados, sino simplemente, que la oración en uso no es un conjunto de trazos y sonidos a los que haya que agregar las condiciones para su aserción. La posibilidad de referir -concluye Putnam- no está sujeta a "técnicas" de uso, sino a capacidades diversas y a actividades lingüísticas y no lingüísticas; no es algo que pueda realizarse de.manera aislada y de una sola vez.

Notemos que no solamente se está haciendo aquí hincapié en el carácter complejo de la articulación de percepción y lenguaje en cuanto funcionan ya como instancias interpretativas, sino también en la función referencial del lenguaje de manera directa; es decir, en lo que interesa para la reafirmación del realismo. Si bien diversos intérpretes de Wittgenstein han identificado el "uso" del cual éste habla con "condiciones de aseveración", presentándolo así como un antirrealista, Putnam insiste en su lectura realista del mismo. Su lectura del parágrafo 95 de las Investigaciones Filosóficas de Wittgenstein se detiene en mostrar que cuando hablamos y significamos que las cosas son de tal o cual forma, también significamos que son asíi ${ }^{30}$ El acento está puesto aquí en afirmar que algo es de tal o cual forma y que tenemos capacidad para pensar en ello, aún más allá de nuestras actuales posibilidades de verificación. Así lo indican la posibilidad de expectativas respecto a situaciones aún no experimentadas, o dicho de otra forma, a lo que "excede"

absurdo de pensar en una imagen mental que reproduzca la ambigüedad del dibujo, sino también la imposibilidad de pensar en la representación como una especie de segundo dibujo mental que tuviese luego que ser interpretado. El "ver como" es ya una interpretación. Cfr. Wittgenstein, Investigaciones filosóficas, 508. Del mismo modo en la comprensión del lenguaje, no captamos primero un conjunto de signos y luego lo interpretamos y luego lo referimos.

${ }^{30}$ Wittgenstein sostiene en el mencionado parágrafo: "Cuando decimos y significamos, que las cosas son así y asá, no nos mantenemos con lo que significamos en algún sitio ante el hecho, sino que significamos que esto es y aquello es asi y asá (das und das-so und so-ist)". 
ciertas condiciones de experiencia ${ }^{31}$. De esta forma se intenta disipar tanto un modo estrecho de llevar a cabo la discusión realismo/irrealismo, establecida sobre los términos en que Dummett lo estructuró, como una concepción limitativa del lenguaje.

\section{Lenguaje y realidad}

\section{¿Por qué poner como eje de la discusión la tesis de la independencia?}

Si bien el desafío planteado inicialmente fue encontrar una tercera vía entre el realismo de tipo metafísico y el "irrealismo", el camino que ha ido adoptando Putnam se aleja cada vez más de una posible posición intermedia. Lo que ofrece no es una conjunción de tesis tomadas de uno y otro, ni una superación dialéctica; su estrategia ha consistido más bien en debilitar la antinomia, en un movimiento "negativo" de desarticulación de supuestos, en los que se incluyen los propios, y en modificar los ejes de la discusión. En sucesivas ocasiones indica que lo importante es discernir lo que puede decirse con sentido. Si atendemos a los análisis precedentes, vemos que no tiene sentido preguntarse por la "independencia" del mundo respecto al lenguaje. Más bien es a la inversa; hay que pensar el lenguaje (como realidad empírica) como parte del mundo, y explicitar su relación con la experiencia.

Se trata de concebir el lenguaje de una manera diferente, pero también de reconsiderar el modo en que se abordan las cuestiones filosóficas a partir del privilegio concedido al análisis del lenguaje. Hemos visto que el realismo interno no fue ajeno a las dificultades del semanticismo; que la apelación a la idea de referencia directa no ha sido suficiente para sostener las intuiciones realistas, y menos aún para dar cuenta de los procesos de corrección en orden a la validez. Si pensamos la relación lenguaje-mundo desde un punto de vista estático, la circularidad es inevitable; no podemos ir más allá del lenguaje. Pero puede pensarse también como un proceso dinámico (una especie de marcha en zig-zag) que permita que el lenguaje pueda ser corregido desde la experiencia.

\footnotetext{
${ }^{31} \mathrm{La}$ insistencia de Dumett en que el objetivo de la filosofía del lenguaje debería especificar recursivamente cómo se pueden verificar los enunciados del lenguaje, hace discutible la idea de lenguaje que sostiene, p.71.
} 
Sin duda esta nueva perspectiva reclama el desarrollo de un programa respecto al alcance de nuestras capacidades, que hasta el momento está solamente perfilado. Más que una solución al respecto, lo que ofrece Putnam son ciertas líneas de investigación, a nuestro juicio, prometedoras. Señalamos especialmente las siguientes cuestiones a explorar:

-La posibilidad de una formación de sentido pre-lingüística. Si la experiencia puede corregir al lenguaje, pareciera que es posible hablar de una cierta separación, de una experiencia no tamizada por el lenguaje. Este es tal vez el punto más álgido, ya que una concepción de la percepción que podríamos llamar "hermenéutica" supone una anticipación de sentido y habría que precisar hasta qué punto esto es independiente del lenguaje. Los textos de esta última etapa, revelan la intención de encontrar una posición equilibrada. Por un lado habrá que considerar el modo en que se presenta la percepción en los casos en que no está la mediación lingüística, como en el caso de los animales, por ejemplo. Putnam llama la atención sobre el hecho de que -aún admitiendo que los procesos cognoscitivos de los animales en distinta medida difieren de la capacidad humana- hay una cierta continuidad, en la medida en que estas capacidades dependen también de una naturaleza biológica ${ }^{32}$.

Pero por otro lado, no podemos dejar de ver que en el caso del hombre el nivel perceptivo no es totalmente separable y que el lenguaje no funciona como un mero instrumento de transmisión. El lenguaje modaliza al pensamiento, alterando incluso el rango de experiencias que podamos tener. Las formas en que extiende las capacidades cognoscitivas son múltiples; por él podemos ir más allá de lo meramente observable, formular refinadas teorías, potenciar el pensamiento. ¿Damos entonces la prioridad al análisis del lenguaje?

En este movimiento zigzagueante, hay que reconocer que el lenguaje no encuentra en sí mismo su sustento; descansa en la primitiva actitud preverbal hacia el mundo. Es "parasitario" de la percepción ${ }^{33}$. El tema tiene especial interés porque nos permitiría avanzar en la cuestión de la posible independencia de la construcción de sentidos respecto al lenguaje. Putnam

\footnotetext{
32 TTC, p. 59.

33 TTC, p. 70.
} 
admite que puede haber algún contenido de experiencia no conceptualizado, y consiguientemente habría que dar lugar a un nivel pre-categorial ${ }^{34}$.

-La otra cuestión es el modo en que la noción de verdad se vincula al realismo y la posibilidad de una verdad pre-epistémica. Aún cuando la concepción epistémica de verdad ha mostrado sus puntos débiles, Putnam sigue manteniendo que no podemos reducir el problema de la verdad al problema de la justificación. Se ha opuesto tenazmente a lo que señala como los errores del verificacionismo y del deflacionismo, y a lo que considera sus consecuencias disolventes respecto a las cuestiones filosóficamente relevantes. El realismo puede sostenerse sólo si se acepta que la verdad es "trascendente" a la justificación ${ }^{35}$.

Se ha dicho anteriormente que lo relevante es interrogarnos acerca de cómo el lenguaje puede "hacer justicia con el mundo", y esto se conecta directamente con la posibilidad de no reducir lo que puede admitirse como verdadero, a lo que puede ser verificado. La alternativa correcta a la concepción de la verdad como propiedad sustantiva, propia del realismo metafísico, no es entonces pensar nuestros enunciados como meras marcas y ruidos que nuestra comunidad nos ha enseñado a asociar con determinadas condiciones de verificación, sino reconocer que los enunciados empíricos, tengan o no la palabra verdad, hacen afirmaciones sobre el mundo. Nuevamente en este caso se apela a la recuperación de aquellas intuiciones básicas subyacentes a nuestro saber. "Si aceptamos que comprender la oración 'Lizzie Boden mató a sus padres con un hacha' no es simplemente el ser capaz de reconocer una verificación en nuestra propia experiencia- es decir, aceptar que somos capaces de concebir como fueron las cosas que no podemos verificar- entonces no aparecerá como tan 'mágico' o 'misterioso' que podamos comprender la afirmación de que esa oración es verdadera. Lo que la hace verdadera, en caso de que lo sea, es sencillamente que Lizzie

34 TTC, p. 184. Putnam ha dado lugar a la posibilidad de una experiencia no conceptualizada; sin embargo, "un contenido epistemológicamente importante como el reconocimiento de algo como un objeto de cierto tipo, es irreductiblemente conceptual. Estas son las experiencias a las que apelamos para la justificación de las creencias. Cuando se habla de justificación, conceptualización y lenguaje son inevitấbles. Sobre percepción y concepción, p. 183-4 y 187.

${ }^{35}$ Discusión con Dummet, Cfr. TTC, p. 60- 69. 
Boden mató a sus padres con un hacha. En este caso, decir que la verdad trasciende a su reconocimiento, no supone nada más que decir que unos asesinatos trascienden a su reconocimiento. $\mathrm{Y}$ ¿pensaríamos alguna vez que se pueden reconocer todos los asesinos? ¿O vamos a considerar que es creer en los poderes mágicos de la mente creer que hay ciertos y determinados individuos que son o fueron asesinos y que no pueden detectarse como tales?"36.

El realismo "natural" se vincula de esta forma a una idea de verdad intuitiva y "pre-epistémica"37. Podemos concebir también aquello que no podemos verificar; y esto tiene que ver con la posibilidad de "extensión" del lenguaje a otros juegos, con la posibilidad de transponer términos e inventar nuevos usos. La corrección o incorrección del discurso varía según el juego en que se dé. Esto significa que nuestra comprensión de lo que llega a ser verdad, en un caso particular, depende de nuestra capacidad para dominar el juego de lenguaje en que se dan y las acciones a las que se vincula. La afirmación de Putnam es explícita: también la verdad tiene una pluralidad de usos, y podemos incluso pensar en nuevos usos que aparecen en la medida en que aparecen nuevas formas de discurso ${ }^{38}$. Una vez más, digamos que esto no es relativismo, sino la alternativa que se ofrece a una concepción que restrictivamente reduce la verdad a lo que puede ser determinado según criterios de verificación.

La admisión de una verdad pre-epistémica indica un posible camino para superar las limitaciones del internalismo, pero de ninguna manera puede considerarse un cierre, sino más bien un paso en otra dirección. Queda aún como tema pendiente la precisión de cómo se conjugarían las instancias de justificación con este nivel pre-epistémico y con la posibilidad de extensión del lenguaje a otros juegos.

\footnotetext{
36 TTC, p. 80.

37 Perona, A.: ‘ ¿Objetividad sin universalidad? El realismo de H. Putnam, en Arenas, L., Muñoz, J. y Perona, A. El retorno del pragmatismo- Madrid, Trotta, 2001, p. 297. ${ }^{38}$ TTC, p. 84.
} 


\title{
Resumen
}

El trabajo se aboca al problema del realismo, como uno de los focos en los que convergen cuestiones semánticas, ontológicas y epistémicas. El análisis parte del realismo interno de Putnam, señalando sus dificultades y el tránsito al realismo pragmático o natural. Este tránsito implica la revisión de la noción epistémica de verdad y de las instancias de justificación, y consiguientemente de las relaciones que se establecen entre la actividad perceptiva y las prácticas lingüísticas.

Palabras clave: "Putnam", "realismo", "verdad"

\begin{abstract}
The work deals with the problem of realism, as one of the focuses in which semantic, ontological and epistemic issues merge together. The analysis starts from Putnam's internal realism, pointing out its difficulties and the movement towards pragmatic or natural realism. This movement implies the reviewing of the epistemic notion of truth and of the instances of justification, and consequently, of the relations established between perceptive activity and linguistic practices.
\end{abstract}

Key Words: "Putnam", “realism”, “truth" 